A N N A LES

UNIVERSITATIS MARIAE CURIE-SKŁODOWSKA

LUBLIN - POLONIA

VOL. LXVI, NO. 1, 2012

SECTIO A

$25-39$

\author{
MICHAEL GIL'
}

\title{
Resolvent and spectrum of a nonselfadjoint differential operator in a Hilbert space
}

\begin{abstract}
We consider a second order regular differential operator whose coefficients are nonselfadjoint bounded operators acting in a Hilbert space. An estimate for the resolvent and a bound for the spectrum are established. An operator is said to be stable if its spectrum lies in the right half-plane. By the obtained bounds, stability and instability conditions are established.
\end{abstract}

1. Introduction. The present paper is devoted to the spectrum localization of regular nonselfadjoint differential operators whose coefficients are bounded operators acting in a separable Hilbert space, and the norm estimates for their resolvents.

The literature on the theory of abstract differential operators is rather rich, but mainly is devoted to the coercitivity of operators and maximal regularity of solutions of the relevant equations, cf. the well-known books $[11,12,14]$ and references therein. At the same time, the spectral theory of differential operators with operator coefficients is not enough developed. The works $[1,2,3,4]$ should be mentioned. In [3] the sum of subtraction of the eigenvalues of two selfadjoint differential operators with unbounded operator coefficient is investigated. In the monograph [2], the Hardy type inequalities for abstract differential operators are established. In the paper

2000 Mathematics Subject Classification. 34K30, 47A10.

Key words and phrases. Abstract differential operator, spectrum, resolvent, stability, instability. 
[1] the authors consider the Sturm-Liouville operator with variable selfadjoint operators in a Hilbert space. That paper extends some classical results of the spectral theory of the Sturm-Liouville operators. In the paper [4], a regularized trace formula for a differential operator of second order with unbounded operator coefficients on a finite interval is established. The monograph [13] considers the interplay between spectral and oscillatory properties of both finite and infinite systems of linear ordinary differential selfadjoint operators. These can be written as single differential equations with matrix-valued and (bounded) operator-valued coefficients, respectively.

The aim of this paper is to generalize the main result from [8] to a nonselfadjoint differential operator with coefficients from a class of bounded operators in a Hilbert space.

Everywhere below $H$ is a separable Hilbert space with a scalar product $(\cdot, \cdot)_{H}$ and the norm $\|\cdot\|_{H}=\sqrt{(\cdot, \cdot)_{H}} . I$ is the unit operator in the corresponding space. All the considered operators are linear. For an operator $T, \sigma(T)$ denotes the spectrum; $R_{\lambda}(T)=(T-\lambda I)^{-1}(\lambda \notin \sigma(T))$ is the resolvent, $T^{*}$ is the adjoint operator, $\|T\|_{H}$ is the operator norm in $H ; \lambda_{j}(T)$ $(j=1,2, \ldots)$ are the eigenvalues of $T$, counted with their algebraic multiplicities; $\rho(T, \lambda):=\inf _{t \in \sigma(T)}|\lambda-t|$ is the distance between $\sigma(T)$ and a complex point $\lambda$.

Let $B(x), C(x)$ be continuous in the operator norm functions defined on a finite real segment $[a, b]$ whose values are bounded operators acting in $H$. The main object of this paper is the differential operator

$$
E=-\frac{d^{2}}{d x^{2}}+2 B(x) \frac{d}{d x}+C(x) \quad(a<x<b)
$$

with the boundary value conditions

$$
y(a) \cos \alpha+y^{\prime}(a) \sin \alpha=0 ; \quad y(b) \cos \beta+y^{\prime}(b) \sin \beta=0
$$

for some $\alpha, \beta \in[0,2 \pi)$. Or $y(a)=y(b) ; y^{\prime}(a)=y^{\prime}(b)$.

Introduce the Hilbert space $L^{2}=L^{2}([a, b], H)$ of functions defined on $[a, b]$ with values in $H$, the scalar product

$$
(y, v)_{L^{2}}:=\int_{a}^{b}(y(x), v(x))_{H} d x
$$

and the norm $\|\cdot\|_{L^{2}}=\sqrt{(\cdot, \cdot)_{L^{2}}}$.

The domain of operator (1.1) is defined as

$$
\begin{aligned}
\operatorname{Dom}(E)=\{ & y \in L^{2}=L^{2}([a, b], H): \frac{d^{2} y(x)}{d x^{2}} \in L^{2}, \\
& \left.y(a) \cos \alpha+y^{\prime}(a) \sin \alpha=0 ; y(b) \cos \beta+y^{\prime}(b) \sin \beta=0\right\},
\end{aligned}
$$

or

$$
\operatorname{Dom}(E)=\left\{y \in L^{2}: \frac{d^{2} y(x)}{d x^{2}} \in L^{2} ; y(a)=y(b), y^{\prime}(a)=y^{\prime}(b)\right\}
$$


2. Auxiliary results. Let $V(x)$ be the Cauchy operator of the equation

$$
u^{\prime}(x)=B(x) u(x),
$$

at a point $x_{0} \in[a, b]$. That is, $u(x)=V(x) u\left(x_{0}\right)$ for any solution $u(t)$ of (2.3) and $V^{\prime}(x)=B(x) V(x)$. So $V\left(x_{0}\right)=I$. We have $V^{-1}(x) u(x)=u\left(x_{0}\right)$. Hence,

$$
\begin{aligned}
0 & =\frac{d}{d x}\left[V^{-1}(x) u(x)\right]=\left[\frac{d}{d x} V^{-1}(x)\right] u(x)+V^{-1}(x) u^{\prime}(x) \\
& =\left[\frac{d}{d x} V^{-1}(x)\right] u(x)+V^{-1}(x) B(x) u(x)
\end{aligned}
$$

Thus

$$
\frac{d}{d x} V^{-1}(x)=-V^{-1}(x) B(x) .
$$

Put

$$
\Phi(x)=V^{-1}(x) C(x) V(x) .
$$

Then

$$
\begin{aligned}
\frac{d}{d x} & \Phi(x)=\left[\frac{d}{d x} V^{-1}(x)\right] C(x) V(x)+V^{-1}(x) C^{\prime}(x) V(x)+V^{-1}(x) C(x) V^{\prime}(x) \\
& =-V^{-1}(x) B(x) C(x) V(x)+V^{-1}(x) C^{\prime}(x) V(x)+V^{-1}(x) C(x) B(x) V(x) \\
& =V^{-1}(x)\left([C(x), B(x)]_{c}+C^{\prime}(x)\right) V(x) .
\end{aligned}
$$

Here $[C(x), B(x)]_{c}:=C(x) B(x)-B(x) C(x)$ is the commutator. But $V\left(x_{0}\right)$ $=I, \Phi\left(x_{0}\right)=C\left(x_{0}\right)$, so

$$
\Phi(x)-C\left(x_{0}\right)=\int_{x_{0}}^{x} \frac{d}{d s} \Phi(s) d s .
$$

We thus have proved:

Lemma 2.1. Let $C(x)$ have the integrable first derivative. Then

$$
V^{-1}(x) C(x) V(x)-C\left(x_{0}\right)=\int_{x_{0}}^{x} V^{-1}(s)\left([C(s), B(s)]_{c}+C^{\prime}(s)\right) V(s) d s .
$$

In particular, if $C$ and $B$ are constant, then $V(x)=e^{B x}$ and

$$
e^{-B x} C e^{B x}=C+\int_{0}^{x} e^{-B s}[C, B]_{c} e^{B s} d s .
$$

Moreover, if $B(x)$ and its integral commute:

$$
B(x) \int_{x_{0}}^{x} B(s) d s=\int_{x_{0}}^{x} B(s) d s B(x),
$$

then

$$
V(x)=\exp \left[\int_{x_{0}}^{x} B(s) d s\right] .
$$


Indeed, put $J(x):=\int_{x_{0}}^{x} B(s) d s$, then

$$
\begin{aligned}
\frac{d}{d x} \exp \left[\int_{x_{0}}^{x} B(s) d s\right] & =\frac{d}{d x} \exp [J(x)]=\frac{d}{d x} \sum_{k=0}^{\infty} \frac{J^{k}(x)}{k !} \\
& =\sum_{k=1}^{\infty} B(x) \frac{J^{k-1}(x)}{(k-1) !}=B(x) \exp \left[\int_{x_{0}}^{x} B(s) d s\right],
\end{aligned}
$$

as claimed. In the case (2.3), by the previous lemma we have

$$
\begin{gathered}
\Phi(x)-B\left(x_{0}\right)=\int_{x_{0}}^{x} \exp \left[-\int_{x_{0}}^{s} B\left(s_{1}\right) d s_{1}\right]\left([C(s), B(s)]_{c}\right. \\
\left.+C^{\prime}(s)\right) \exp \left[\int_{x_{0}}^{s} B\left(s_{1}\right) d s_{1}\right] d s .
\end{gathered}
$$

If $B(x)$ is selfcommuting: $B(x) B(s)=B(s) B(x)$, then (2.3) and therefore, (2.4) hold. If $B(x)$ and $C(x)$ commute, then

$$
\Phi(x)-C\left(x_{0}\right)=\int_{x_{0}}^{x} V^{-1}(s) C^{\prime}(s) V(s) d s .
$$

3. The autonomous problem. We will investigate operator $E$ as a perturbation of the operator

$$
E_{0}=-\frac{d^{2}}{d x^{2}}+M \quad(0<x<b)
$$

with a constant bounded operator $M$ acting in $H$. Besides the domain of $E_{0}$ is $\operatorname{Dom}(E)$. By $a_{j}=\lambda_{j}(S), j=1,2, \ldots$ we denote the eigenvalues of the operator $S:=-d^{2} / d x^{2}$ defined on $\operatorname{Dom}(E)$. Let $P_{j}$ be the orthogonal eigenprojections of $S$, defined by

$$
P_{j}=-\frac{1}{2 \pi i} \int_{\left|z-a_{j}\right|=\epsilon}(S-z I)^{-1} d z
$$

for a sufficiently small $\epsilon>0$. Then

$$
S=\sum_{j=1}^{\infty} a_{j} P_{j} ; M P_{j}=P_{j} M \text { and } E_{0}=\sum_{j=1}^{\infty}\left(a_{j} I+M\right) P_{j} .
$$

Hence,

$$
R_{\lambda}\left(E_{0}\right)=\sum_{j=1}^{\infty}\left(M+\left(a_{j}-\lambda\right) I\right)^{-1} P_{j}
$$

and

$$
\left\|R_{\lambda}\left(E_{0}\right)\right\|_{L^{2}} \leq \sup _{j}\left\|\left(M+\left(a_{j}-\lambda\right) I\right)^{-1}\right\|_{H} .
$$


It is assumed that there is a continuous monotonically increasing function $F_{M}$, satisfying $F_{M}(0)=0, F_{M}(\infty)=\infty$, such that

$$
\left\|(M-\lambda I)^{-1}\right\|_{H} \leq F_{M}(1 / \rho(M, \lambda)) .
$$

Then $\left\|\left(M+\left(a_{j}-\lambda\right) I\right)^{-1}\right\|_{H} \leq F_{M}\left(1 / \rho\left(M, \lambda-a_{j}\right)\right)$. But $\inf _{j} \rho(M, \lambda-$ $\left.a_{j}\right)=\rho\left(E_{0}, \lambda\right)$. Here $\rho\left(E_{0}, \lambda\right)=\inf _{t \in \sigma\left(E_{0}\right)}|\lambda-t|=\inf _{s \in \sigma(M), j}\left|s+a_{j}-\lambda\right|$. Consequently, by (3.2) we obtain:

Lemma 3.1. Let the conditions (3.3) and

$$
\lambda \neq a_{j}+s \quad(j=1,2, \ldots ; s \in \sigma(S))
$$

hold. Then $\left\|R_{\lambda}\left(E_{0}\right)\right\|_{L^{2}} \leq F_{M}\left(1 / \rho\left(E_{0}, \lambda\right)\right)$.

4. The basic lemma. Let $V(x)$ be the Cauchy operator of equation (2.1) at a point $x_{0}$, again. Put

$$
Z(x)=-B^{\prime}(x)+B^{2}(x)+C(x)
$$

and on $\operatorname{Dom}(E)$ introduce the operator $T$ by

$$
(T f)(x):=f^{\prime \prime}(x)+V^{-1}(x) Z(x) V(x) f(x) \quad(f \in \operatorname{Dom}(E)) .
$$

Lemma 4.1. Let $B(x)$ have a bounded measurable derivative. Then operator $E$ is similar to T. Namely,

$$
E=V(\cdot) T V^{-1}(\cdot)
$$

where $(V(\cdot) f)(x)=V(x) f(x)$.

Proof. Putting $\lambda I=\lambda$, consider the equation

$$
(E-\lambda) y=-\frac{d^{2}}{d x^{2}} y+2 B(x) \frac{d y(x)}{d x}+(C(x)-\lambda) y=f(x) \quad(a<x<b)
$$

with $f \in L^{2}(H)$. Substitute $y=V(x) v$. Then

$$
\begin{aligned}
-\left[V^{\prime \prime}(x) v+2 V^{\prime}(x) v^{\prime}+V(x) v^{\prime \prime}\right]+2 B(x) & {\left[V^{\prime}(x) v+V(x) v^{\prime}\right] } \\
& +(C(x)-\lambda) V(x) v=f(x) .
\end{aligned}
$$

Note that

$$
V^{\prime \prime}(x)=(B(x) V(x))^{\prime}=B^{\prime}(x) V(x)+B(x) V^{\prime}(x)=B^{\prime}(x) V(x)+B^{2}(x) V(x) .
$$

Hence,

$$
\begin{aligned}
-\left[B^{\prime}(x) V(x) v+\right. & \left.B^{2}(x) V(x) v+2 B(x) V(x) v^{\prime}+V(x) v^{\prime \prime}\right] \\
& +2 B(x)\left[B(x) v+V(x) v^{\prime}\right]+C(x) V(x) v-\lambda V(x) v=f(x) .
\end{aligned}
$$

Thus

$$
\begin{aligned}
-V(x) v^{\prime \prime}+\left(-B^{\prime}(x)+\right. & \left.B^{2}(x)+C(x)-\lambda\right) V(x) v \\
& =-V(x) v^{\prime \prime}+(Z(x)-\lambda) V(x) v=f(x) .
\end{aligned}
$$


Therefore,

$$
-v^{\prime \prime}+V^{-1}(\cdot) Z(\cdot) V(\cdot) v-\lambda v=(T-\lambda) v=V^{-1}(\cdot) f .
$$

Hence, $v=R_{\lambda}(T) V^{-1}(\cdot) f$ and consequently,

$$
R_{\lambda}(E) f=y=V(\cdot) v=V(\cdot) R_{\lambda}(T) V^{-1}(\cdot) .
$$

This proves the lemma.

By the previous lemma the spectrum of $E$ and $T$ coincide. Moreover,

$$
\left\|R_{\lambda}(E)\right\|_{L^{2}} \leq m_{0}\left\|R_{\lambda}(T)\right\|_{L^{2}},
$$

where $m_{0}:=\sup _{x \in[a, b]}\|V(x)\|_{H} \sup _{x \in[a, b]}\left\|V^{-1}(x)\right\|_{H}$.

5. The main result. Recall that $Z(x)=-B^{\prime}(x)+B^{2}(x)+C(x)$ and for a fixed $x_{0} \in[a, b]$, put

$$
M=Z\left(x_{0}\right)=-B^{\prime}\left(x_{0}\right)+B^{2}\left(x_{0}\right)+C\left(x_{0}\right)
$$

and

Note that

$$
q:=\sup _{x}\left\|\int_{x_{0}}^{x} V^{-1}(s)\left([Z(s), B(s)]_{c}+Z^{\prime}(s)\right) V(s) d s\right\|_{H} .
$$

$$
[Z(x), B(x)]_{c}=-\left[B^{\prime}(x), B(x)\right]_{c}+[C(x), B(x)]_{c}
$$

and

$$
\frac{d Z(x)}{d x}=-B^{\prime \prime}(x)+B^{\prime}(x) B(x)+B(x) B^{\prime}(x)+C^{\prime}(x) .
$$

Now we are in a position to formulate the main result of the paper.

Theorem 5.1. Let $B(x)$ have an integrable second derivative, $C(x)$ have an integrable first derivative and $M$ be defined by (5.1). Assume that the conditions (3.3) and

$$
q F_{M}\left(1 / \rho\left(E_{0}, \lambda\right)\right)<1
$$

hold. Then $\lambda$ is regular for $E$, and

$$
\left\|(E-\lambda I)^{-1}\right\|_{L^{2}} \leq \frac{m_{0} F_{M}\left(1 / \rho\left(E_{0}, \lambda\right)\right)}{1-q F_{M}\left(1 / \rho\left(E_{0}, \lambda\right)\right)} .
$$

Proof. Thanks to Lemma 2.1,

$$
\begin{aligned}
& \left\|V^{-1}(x) Z(x) V(x)-Z\left(x_{0}\right)\right\|_{H} \\
& \quad=\left\|\int_{x_{0}}^{x} V^{-1}(s)\left([Z(s), B(s)]_{c}+Z^{\prime}(s)\right) V(s) d s\right\|_{H} \leq q
\end{aligned}
$$

for all $x \in[a, b]$. So

$$
\left\|T-E_{0}\right\|_{L^{2}}=\sup _{x}\left\|V^{-1}(x) Z(x) V^{-1}(x)-Z\left(x_{0}\right)\right\|_{H}=q .
$$


By the Hilbert identity for resolvents,

$$
(T-\lambda I)^{-1}-\left(E_{0}-\lambda I\right)^{-1}=(T-\lambda I)^{-1}\left(T-E_{0}\right)\left(E_{0}-\lambda I\right)^{-1} .
$$

Thus, if the inequality

$$
q\left\|\left(\lambda I-E_{0}\right)^{-1}\right\|_{L^{2}}<1
$$

holds, then $\lambda$ is regular for $T$, and

$$
\left\|(T-\lambda I)^{-1}\right\|_{L^{2}} \leq\left\|\left(E_{0}-\lambda I\right)^{-1}\right\|_{L^{2}}\left(1-q\left\|\left(I \lambda-E_{0}\right)^{-1}\right\|_{L^{2}}\right)^{-1} .
$$

Now Lemma 3.1 and (4.3) imply the required result.

There exist various estimates for the Cauchy operator. For instance, recall the Wintner inequalities

$$
e^{\int_{x_{0}}^{x} \beta_{R}(v) d v} \leq \frac{\|V(x) h\|_{H}}{\|h\|_{H}} \leq e^{\int_{x_{0}}^{x} \alpha_{R}(v) d v}
$$

where $\alpha_{R}(x)$ and $\beta_{R}(x)$ are the largest and the smallest eigenvalues, respectively, of the operator $B_{R}(x)=\left(B(x)+B^{*}(x)\right) / 2$, e.g. [5, Theorem III.4.7]. Thus

$$
m_{0} \leq \hat{m}_{R} \text { where } \hat{m}_{R}:=\sup _{x \in[a, b]} e^{-\int_{x_{0}}^{x} \beta_{R}(v) d v} \sup _{x \in[a, b]} e^{\int_{x_{0}}^{x} \alpha_{R}(v) d v}
$$

Hence,

$$
q \leq \hat{q} \text { where } \hat{q}:=\hat{m}_{R} \int_{a}^{b}\left\|[Z(s), B(s)]_{c}+Z^{\prime}(s)\right\|_{H} d s
$$

6. Bounds for the spectrum. Thanks to Theorem 5.1, any $\mu \in \sigma(E)$ satisfies the inequality $q F_{M}\left(E_{0}, \mu\right) \geq 1$. In other words, for any $\mu \in \sigma(E)$, there is an $s \in \sigma\left(E_{0}\right)$, such that

$$
q F_{M}(1 /|\mu-s|) \geq 1 \text {. }
$$

Taking into account the monotonicity of the left-hand part of this inequality we get $\left|\mu-\lambda\left(E_{0}\right)\right| \leq r(q)$, where $r(q)$ is the unique positive root of the equation

$$
q F_{M}(1 / x)=1
$$

We thus have proved the following:

Theorem 6.1. Let $B(x)$ and $C(x)$ satisfy the hypothesis of Theorem 5.1. Let operator $M$ be defined by (5.1). Then for any $\mu \in \sigma(E)$, there are integers $j \geq 1$ and $s \in \sigma(M)$, such that $\left|s+a_{j}-\mu\right| \leq r(q)$, where $r(q)$ is the unique positive root of equation (6.1). 
Let $A$ and $\tilde{A}$ be two linear operators. Then

$$
s v_{A}(\tilde{A}):=\sup _{s \in \sigma(\tilde{A})} \inf _{\mu \in \sigma(A)}|s-\mu|
$$

will be called the spectral variation of $\tilde{A}$ with respect to $A$.

So Theorem 6.1 means that $s v_{E_{0}}(E) \leq r(q)$.

Corollary 6.2. Under the hypothesis of Theorem 5.1, the spectrum of $E$ lies in the union of the sets

$$
\left\{z \in \mathbb{C}:\left|z-s(M)-a_{j}\right| \leq r(q)\right\},
$$

$s \in \sigma(M) ; j=1,2, \ldots$

Operator $E$ is said to be stable if $\operatorname{Re} \sigma(A)>0 . \quad E$ is unstable if $\inf \operatorname{Re} \sigma(A)<0$.

Now Theorem 6.1 implies

Corollary 6.3. Under the hypothesis of Theorem 5.1, let

$$
\inf _{j=1,2, \ldots} a_{j}+\inf \operatorname{Re} \sigma(M)>r(q) .
$$

Then $E$ is stable. Conversely, if $\inf _{j=1,2, \ldots} a_{j}+\inf \operatorname{Re} \sigma(M)<-r(q)$, then $E$ is unstable.

7. Operators admitting triangular representations. For a compact operator $K$ in $H, s_{k}(A), k=1,2, \ldots$, are the singular numbers taken with their multiplicities and enumerated in the decreasing order. By $S N_{r}$ $(1 \leq r<\infty)$, the Schatten-von Neumann ideal is denoted. That is, the ideal of compact operators $K$ in $H$ with the finite norm $N_{r}(K):=$ [Trace $\left.\left(K^{*} K\right)^{r / 2}\right]^{1 / r}$.

Let $c_{0}$ be the space of all scalar sequences tending to zero, $\hat{c}$ be the set of all sequences from $c_{0}$ having a finite number of nonzero entries. A real function $F(h)=F\left(h_{1}, h_{2}, \ldots\right)$ defined on $c_{0}$ is called a norming function, if it satisfies the following conditions: $F(h)>0, h \neq 0 ; F(a h)=|a| F(h)(a \in \mathbb{C})$ and $F(h+w) \leq F(h)+F(w)$ for all $h=\left\{h_{k}\right\}, w=\left\{w_{k}\right\} \in \hat{c}$. In addition, $F(1,0,0, \ldots)=1$. A norming function $F$ is called a symmetrically norming function, if $F\left(h_{1}, h_{2}, \ldots, h_{n}, 0,0, \ldots\right)=F\left(\left|h_{1}\right|,\left|h_{2}\right|, \ldots,\left|h_{n}\right|, 0,0, \ldots\right)$. The following relations are well-known [9, Section 3.3]: if for $h=\left\{h_{k}\right\}, w=$ $\left\{w_{k}\right\} \in \hat{c}$, the inequalities $\left|h_{k}\right| \leq\left|w_{k}\right|$ hold, then $F(h) \leq F(w)$. Moreover, if

$$
w_{1} \geq w_{2} \geq \cdots \geq 0 ; h_{1} \geq h_{2} \geq \cdots \geq 0 \text { and } \sum_{k=1}^{j} w_{k} \leq \sum_{k=1}^{j} h_{k},
$$

then $F(w) \leq F(h)$ for any symmetrically norming function $F$. Furthermore, on the set of all compact operators $K$ acting in $H$, introduce the norm $N_{W}(K)$ by

$$
N_{W}(K)=F\left(s_{1}(K), s_{2}(K), \ldots\right) .
$$


Then the set $W=W(F)$ of operators with the finite norm $N_{W}$ is a symmetrically normed ideal, e.g. [9, Section 3.4].

Let $E(t)$ be a left-continuous orthogonal resolution of the identity in $H$, defined on a real segment $[a, b] . E$ is called a maximal resolution of the identity (m.r.i.), if its every gap $E\left(t_{0}+0\right)-E\left(t_{0}\right)$ (if it exists) is onedimensional, cf. [6].

A compact quasinilpotent operator will be called a Volterra operator. We will say that an m.r.i. $E(t)$ belongs to $A$ (or $A$ has an m.r.i. $E(t)$ ), if $E(t) A E(t)=A E(t)(t \in[a, b])$. We will say that $A$ is a E-triangular operator if it has an m.r.i. $E$ defined on $[a, b]$ and admits the representation

$$
A=D+V
$$

where $D$ is a normal operator and $V$ is a Volterra one, having the following properties:

$$
E(t) V E(t)=V E(t) \text { and } D E(t)=E(t) D \quad(t \in[a, b]) .
$$

An E-triangular operator $A$ has the property

$$
\sigma(A)=\sigma(D),
$$

cf. [6, Lemma 7.5.1]. Each compact operator is E-triangular [9] and each operator having the Schatten-von Neumann Hermitian component is Etriangular; for more details see [6, Chapter 7]. We will call $D$ and $V$ the diagonal and nilpotent part of $A$, respectively.

Lemma 7.1. Let $A$ be an E-triangular operator whose nilpotent part $V$ belongs to a symmetrically normed ideal $W$. In addition, let

$$
\left\|(I-V)^{-1}\right\| \leq \phi\left(N_{W}(V)\right),
$$

where $\phi(x), x>0$ is a nondecreasing continuous function. Then

$$
\left\|R_{\lambda}(A)\right\| \leq \frac{1}{\rho(A, \lambda)} \phi\left(\frac{N_{W}(V)}{\rho(A, \lambda)}\right) \quad(\lambda \notin \sigma(A)) .
$$

Proof. Let $\lambda$ be a regular point of the operator $D$. According to the triangular representation (7.1), we obtain

$$
R_{\lambda}(A)=(D+V-\lambda I)^{-1}=R_{\lambda}(D)\left(I+V R_{\lambda}(D)\right)^{-1} .
$$

Operator $V R_{\lambda}(D)$ for a regular point $\lambda$ of the operator $D$ is a Volterra one due to Lemma 7.3.4 from [6]. Therefore,

$$
\left.\left\|R_{\lambda}(A)\right\|=\| R_{\lambda}(D)\left(I-V R_{\lambda}(D)\right)\right)^{-1}\|\leq\| R_{\lambda}(D) \| \phi\left(N_{W}\left(V R_{\lambda}(D)\right)\right) .
$$

But $N_{W}\left(V R_{\lambda}(D)\right) \leq\left\|R_{\lambda}(D)\right\| N_{W}(V)$ and $\left\|R_{\lambda}(D)\right\|=1 / \rho(A, \lambda)$. This proves the result. 
If $\phi(0)=1$, then the previous lemma is sharp: if $A$ is a normal operator, then $V=0$ and inequality (7.5) becomes the equality $\left\|R_{\lambda}(A)\right\|=\frac{1}{\rho(A, \lambda)}$. By the Weyl inequalities,

$$
N_{W}(D)=F\left(\lambda_{1}(A), \lambda_{2}(A), \ldots\right) \leq F\left(s_{1}(A), s_{2}(A), \ldots\right)=N_{W}(A) .
$$

We thus have

$$
N_{W}(V) \leq N_{W}(D)+N_{W}(A) \leq 2 N_{W}(A) .
$$

Now the previous lemma implies:

Corollary 7.2. Let $A \in W$ and condition (7.4) hold. Then

$$
\left\|R_{\lambda}(A)\right\| \leq \frac{1}{\rho(A, \lambda)} \phi\left(\frac{2 N_{W}(A)}{\rho(A, \lambda)}\right) \quad(\lambda \notin \sigma(A)) .
$$

8. Operators with Schatten-von Neumann components. In this section we illustrate the results of the previous section in the case

$$
A_{I, t}:=\left(e^{i t} A-e^{-i t} A^{*}\right) / 2 i \in S N_{p} \quad(p=1,2, \ldots)
$$

for a $t \in[0,2 \pi]$. To this end we need the following result.

Lemma 8.1. Let $V \in S N_{1}$ be a quasinilpotent operator. Then

$$
\left\|(I-V)^{-1}\right\|^{2} \leq \prod_{k=1}^{\infty}\left(1+s_{k}\left(2 V_{R}-V^{*} V\right)\right) .
$$

Here $s_{k}\left(2 V_{R}-V^{*} V\right)$ are the singular values of $2 V_{R}-V^{*} V$ counted with their multiplicities and enumerated in the decreasing way.

Proof. We have $\operatorname{det}(I-V)\left(I-V^{*}\right)=\operatorname{det}(I-V) \operatorname{det}\left(I-V^{*}\right)=1$. So

$$
1=\operatorname{det}(I-V)\left(I-V^{*}\right)=\operatorname{det}(I-T), \text { where } T=2 V_{R}-V V^{*} .
$$

Let $\lambda_{k}=\lambda_{k}(T)$. Then $\left|\lambda_{k}\right|=s_{k}(T)=s_{k}\left(2 V_{R}-V V^{*}\right)$. Assume that $\min _{k}\left|1-\lambda_{k}\right|=\left|1-\lambda_{j}\right|$ for an index $j$. Then $\left\|(I-V)^{-1}\right\|^{2}=\left|1-\lambda_{j}\right|^{-1}$. So

$$
1=|\operatorname{det}(I-T)|=\left\|(I-V)^{-1}\right\|^{-2} \prod_{k=1, k \neq j}^{\infty}\left|1-\lambda_{k}\right|
$$

and thus,

$$
\left\|(I-V)^{-1}\right\|^{2}=\prod_{k=1, k \neq j}^{\infty}\left|1-\lambda_{k}\right| \leq \prod_{k=1}^{\infty}\left(1+\left|\lambda_{k}\right|\right),
$$

as claimed.

Introduce the function

$$
\Phi\left(t_{1}, t_{2}, \ldots, t_{j}\right)=\prod_{k=1}^{\infty}\left(1+t_{k}\right) \quad\left(t_{1}>t_{2}>\cdots>t_{j} \geq 0\right) .
$$


Clearly,

But

$$
\frac{\partial \Phi}{\partial t_{k}}=\frac{\Phi}{1+t_{k}}>\frac{\partial \Phi}{\partial t_{k+1}} \geq 0
$$

$$
\sum_{k=1}^{j} s_{k}\left(2 V_{R}-V^{*} V\right) \leq \sum_{k=1}^{j} s_{k}\left(2 V_{R}\right)+s_{k}\left(V^{*} V\right) .
$$

Therefore, by Lemma 2.7.5 [9],

$$
\prod_{k=1}^{\infty}\left(1+s_{k}\left(2 V_{R}-V^{*} V\right)\right) \leq \prod_{k=1}^{\infty}\left(1+2 s_{k}\left(V_{R}\right)+s_{k}^{2}(V)\right)=\prod_{k=1}^{\infty}\left(1+s_{k}(V)\right)^{2}
$$

Now the latter lemma yields our next result.

Corollary 8.2. Let $V \in S N_{1}$ be a quasinilpotent operator. Then

$$
\left\|(I-V)^{-1}\right\| \leq \prod_{k=1}^{\infty}\left(1+s_{k}(V)\right)
$$

Note that inequality (8.3) is a particular case of the well-known Theorem V.5.1 from [9], but we suggest a considerably new approach. Besides, the previous lemma is sharper than (8.3). Furthermore, clearly,

$$
(I-V)^{-1}=\left(I+V+\cdots+V^{p-1}\right)\left(I-V^{p}\right)^{-1}
$$

for any positive integer $p$. Now Corollary 8.2 implies:

Lemma 8.3. Let $V \in S N_{p}(p=1,2, \ldots)$ be a quasinilpotent operator. Then

$$
\left\|(I-V)^{-1}\right\| \leq\left\|I+V+\cdots+V^{p-1}\right\| \prod_{k=1}^{\infty}\left(1+s_{k}^{p}(V)\right) \leq \psi_{p}\left(N_{p}(V)\right),
$$

where

$$
\psi_{p}(x):=e^{x^{p}} \sum_{k=0}^{p-1} x^{k} \quad(x \geq 0) .
$$

This result and Lemma 7.1 yield the next result.

Corollary 8.4. Let $A$ be an E-triangular operator whose nilpotent part $V \in S N_{p}(p=1,2, \ldots)$. Then

$$
\left\|R_{\lambda}(A)\right\| \leq \frac{1}{\rho(A, \lambda)} \psi_{p}\left(\frac{N_{p}(V)}{\rho(A, \lambda)}\right) \quad(\lambda \notin \sigma(A)) .
$$

As it is proved in Theorems III.6.2 and III.6.3 [10], if $V_{I} \in S N_{p}$, then $N_{p}(V) \leq \tilde{b}_{p} N_{p}\left(V_{I}\right)$, where $\tilde{b}_{p}$ is a constant dependent only on $p$, and $\tilde{b}_{p} \leq$ $1+\frac{p}{e^{2 / 3} \ln 2}$. Besides, $\tilde{b}_{2}=\sqrt{2}$. Since $N_{p}(V)=N_{p}\left(e^{i t} V\right)$ for a real $t$, we have

$$
N_{p}(V) \leq \tilde{b}_{p} N_{p}\left(V_{I, t}\right) \text {. }
$$


But by the Weyl inequalities [9], $N_{p}\left(D_{I, t}\right) \leq N_{p}\left(A_{I, t}\right)$ and therefore,

$$
N_{p}(V) \leq \tilde{b}_{p} N_{p}\left(V_{I, t}\right) \leq \tilde{b}_{p} N_{p}\left(A_{I, t}\right)+\tilde{b}_{p} N_{p}\left(D_{I, t}\right) \leq 2 \tilde{b}_{p} N_{p}\left(A_{I, t}\right) .
$$

Thanks to Theorem 7.6.1 [6], under condition (8.1), $A$ is an $E$-triangular operator. Now Corollary 8.4 implies the following result.

Theorem 8.5. Let condition (8.1) hold. Then

$$
\|R(A)\| \leq \frac{1}{\rho(A, \lambda)} \psi_{p}\left(\frac{b_{p} N_{p}\left(A_{I, t}\right)}{\rho(A, \lambda)}\right) \quad\left(b_{p}=2 \tilde{b}_{p}, \lambda \notin \sigma(A)\right) .
$$

Remark 8.6. In the case $A \in S N_{p}, p \geq 1$, we have $N_{p}(D) \leq N_{p}(A)$ and therefore, $N_{p}(V) \leq 2 N_{p}(A)$. Thus in (8.7) one can replace $b_{p} N_{p}\left(A_{I, t}\right)$ by $2 N_{p}(A)$.

Note that (8.7) is a generalization of Theorem 7.7.1 [6], which is proved in the case $A-A^{*} \in S N_{2 r}, r=1,2, \ldots$.

In the case $M_{I, t}=\left(M e^{i t}-e^{-i t} M^{*}\right) / 2 i \in S N_{p}, 1<p<\infty$, according to Theorem 8.5, equation (6.1) takes the form

$$
\frac{q_{0}}{x} \psi_{p}\left(\frac{b_{p} N_{p}\left(M_{I, t}\right)}{x}\right)=1
$$

To estimate the roots of this equation we need the following result.

Lemma 8.7. For any integer $p \geq 1$, the unique positive root $z_{a}$ of the equation

$$
\sum_{j=0}^{p-1} \frac{1}{y^{j+1}} \exp \left[\frac{1}{y^{p}}\right]=a \quad(a=\text { const }>0)
$$

satisfies the inequality $z_{a} \leq \delta_{p}(a)$, where

$$
\delta_{p}(a):= \begin{cases}p e / a & \text { if } a \leq p e, \\ \sqrt[p]{2}[\ln (a e / p)]^{-1 / p} & \text { if } a>p e .\end{cases}
$$

Proof. Let

$$
p e \geq a \text {. }
$$

Since the function

$$
f(y)=\sum_{j=0}^{p-1} \frac{1}{y^{j+1}} \exp \left[\frac{1}{y^{p}}\right]
$$

is nonincreasing and $f(1)=p e$, we have $z_{a} \geq 1$. But because of (8.9),

$$
z_{a}=\frac{1}{a} \sum_{j=0}^{p-1} \frac{1}{z_{a}^{j}} \exp \left[z_{a}^{-p}\right] \leq \frac{p e}{a} .
$$


So in the case (8.10), the lemma is proved. Now let $p e<a$. Then $z_{a} \leq 1$. But

$$
\sum_{j=0}^{p-1} x^{j+1} \leq p x^{p} \leq p \exp \left[x^{p}-1\right] \quad(x \geq 1)
$$

So

$$
f(y)=\sum_{j=0}^{p-1} \frac{1}{y^{j+1}} \exp \left[\frac{1}{y^{p}}\right] \leq p e^{-1} \exp \left[\frac{2}{y^{p}}\right] \quad(y \leq 1) .
$$

We thus have $a=f\left(z_{a}\right) \leq p e^{-1} \exp \left[\frac{2}{z_{a}^{p}}\right]$. This finishes the proof.

Put in (8.8) $y=\frac{x}{b_{p} N_{p}\left(M_{I, t}\right)}$. Then we obtain (8.9) with $a=\gamma(q)$ with

$$
\gamma(q):=\frac{b_{p} N_{p}\left(M_{I, t}\right)}{q_{0}}
$$

Hence, a unique positive root $r(q)$ of (8.8) satisfies the inequality

$$
r(q) \leq \delta(q) \text { where } \delta(q):= \begin{cases}p e / \gamma(q) & \text { if } \gamma(q) \leq p e \\ \sqrt[p]{2}[\ln (\gamma(q) e / p)]^{-1 / p} & \text { if } \gamma(q)>p e\end{cases}
$$

Now Theorem 6.1 implies:

Corollary 8.8. Let $B(x)$ and $C(x)$ satisfy the hypothesis of Theorem 5.1 and $M_{I, t} \in S N_{p}(t \in[0,2 \pi] ; 1<p<\infty)$. Then for any $\mu \in \sigma(E)$, there is an integer $j \geq 1$ and an $s \in \sigma(M)$, such that $\left|s+a_{j}-\mu\right| \leq r(q) \leq \delta(q)$, where $r(q)$ is the unique positive root of equation (8.8) and $\delta(q)$ is defined by $(8.11)$.

In particular, if $\inf _{j=1,2, \ldots} a_{j}+\inf \operatorname{Re} \sigma(M)>\delta(q)$, then $E$ is stable. Conversely, if $\inf _{j=1,2, \ldots} a_{j}+\inf \operatorname{Re} \sigma(M)<-\delta(q)$, then $E$ is unstable.

9. Example. Let $H=L^{2}\left([-\pi, \pi], \mathbb{C}^{n}\right)$ be a Hilbert space of functions defined on $[-\pi, \pi]$ with values in a complex Euclidean space $\mathbb{C}^{n}$ and equipped with the scalar product

$$
(f, h)_{H}=\int_{-\pi}^{\pi}(f(s), h(s))_{C^{n}} d s,
$$

where $(\cdot, \cdot)_{C^{n}}$ is the scalar product in $\mathbb{C}^{n}$. In addition, $\|\cdot\|_{C^{n}}=\sqrt{(\cdot, \cdot)_{C^{n}}}$. Consider the Dirichlet problem

$$
E u=-\frac{\partial^{2} u(x, y)}{\partial x^{2}}+b(x) \frac{\partial u(x, y)}{\partial x}+\int_{-\pi}^{\pi} K_{0}(x-s) u(x, s) d s
$$

$(x \in(0,1), y \in[-\pi, \pi])$,

$$
u(0, y)=u(1, y)=0
$$

$(y \in[-\pi, \pi])$, where $b(x)$ is a real twice continuously differentiable scalar function, and $K_{0}$ is a matrix-valued function defined on $[-\pi, \pi]$ with $\left\|K_{0}(x)\right\|_{C^{n}} \in L^{2}([-\pi, \pi], \mathbb{C})$. Here $\|A\|_{C^{n}}$ means the operator (spectral) 
norm of an $n \times n$-matrix $A$. In the considered case $a_{j}=j^{2}, B(x) u=b(x) u$, and

$$
C(x) u(x, y)=C_{0} u(x, y)=\int_{-\pi}^{\pi} K(y-s) u(x, s) d s
$$

does not depend on $x$. Let

$$
K_{0}(y)=\frac{1}{2 \pi} \sum_{k=-\infty}^{\infty} \tau_{k} e^{i k y}
$$

be the Fourier expansion with the matrix Fourier coefficients

$$
\tau_{k}=\int_{-\pi}^{\pi} K_{0}(s) e^{-i k s} d s .
$$

We have $C_{0} e^{i k x}=\tau_{k} e^{i k x}$. Let $d_{j k}$ be an eigenvector of $\tau_{k}$, corresponding to an eigenvalue $\lambda_{j}\left(\tau_{k}\right)(j=1, \ldots, n)$. Then

$$
C_{0} e^{i k x} d_{j k}=\int_{-\pi}^{\pi} K_{0}(x-s) d_{j k} e^{i k s} d s=e^{i k x} \tau_{k} d_{j k}=e^{i k x} \lambda_{j}\left(\tau_{k}\right) d_{j k} .
$$

So the spectrum of $C_{0}$ consists of the points

$$
\lambda_{j}\left(\tau_{k}\right) \quad(k=0, \pm 1, \pm 2, \ldots ; j=1, \ldots, n) .
$$

In addition, $Z(x)=-B^{\prime}(x)+B^{2}(x)+C(x)=-b^{\prime}(x)+b^{2}(x)+C_{0}$. Hence, $[Z(x), B(x)]_{c}=0$, and $\left\|Z^{\prime}(x)\right\|_{H}=\sqrt{2 \pi}\left|b^{\prime \prime}(x)-2 b(x) b^{\prime}(x)\right|$. Take $M=$ $Z(0)=-b^{\prime}(0)+b^{2}(0)+C_{0}$. Then the spectrum of $M$ consists of the points

$$
\lambda_{j k}(M)=b^{\prime}(0)+b^{2}(0)+\lambda_{j}\left(\tau_{k}\right) \quad(k=0, \pm 1, \pm 2, \ldots ; j=1, \ldots, n) .
$$

Furthermore, since $C_{0}$ and $B(x)$ commute, we have $m_{0}=1$. By (5.8)

$$
q \leq \hat{q}:=\int_{0}^{1}\left\|[Z(s), B(s)]_{c}+Z^{\prime}(s)\right\|_{H} d s=\int_{0}^{1}\left|b^{\prime \prime}(x)-2 b(x) b^{\prime}(x)\right| d x .
$$

Moreover,

$$
N_{2}^{2}\left(M_{I}\right)=\int_{-\pi}^{\pi} \int_{-\pi}^{\pi} N_{2}^{2}\left(K_{0 I}(x-s)\right) d x d s
$$

where $M_{I}, K_{0 I}(x)$ are the imaginary Hermitian components of $M$ and $K_{0}(x)$, respectively. Thanks to Corollary 8.8, the spectrum of problem (9.1), (9.2) lies in the union of the sets

$$
\left\{z \in \mathbb{C}:\left|z-\lambda_{j k}(M)-m^{2}\right| \leq r(\hat{q})\right\}
$$

$(j=1,2, \ldots, n ; k=0, \pm 1, \pm 2, \ldots ; m=1,2, \ldots)$. Here $r(\hat{q})$ is the unique positive root of equation (8.8) with $p=2, b_{2}=\sqrt{2}$ and $q=\hat{q}$. Besides, the bound (8.11) is valid.

In particular, if $1+\inf _{j, k} \operatorname{Re} \lambda_{j, k}(M)>r(\hat{q})$, then $E$ is stable. If $1+$ $\inf _{j, k} \operatorname{Re} \lambda_{j, k}(M)<-\hat{q}$, then it is unstable. 


\section{REFERENCES}

[1] Adiguzelov, E., Karayel, S., A selfadjoint expansion of a symmetric differential operator with operator coefficient, Int. J. Contemp. Math. Sci. 2 (2007), no. 21-24, 1053-1067.

[2] Amrein, W., Boutet de Monvel-Berthier, A. and Georgescu, V., Hardy type inequalities for abstract differential operators, Mem. Amer. Math. Soc. 70 (1987), no. 375, $119 \mathrm{pp}$.

[3] Baksi, O., Sezer, Y. and Karayel, S., The sum of subtraction of the eigenvalues of two selfadjoint differential operators with unbounded operator coefficient, Int. J. Pure Appl. Math. 63 (2010), no. 3, 255-268.

[4] Gül, E., A regularized trace formula for a differential operator of second order with unbounded operator coefficients given in a finite interval, Int. J. Pure Appl. Math. 32 (2006), no. 2, 225-244.

[5] Daleckii, Yu L., Krein, M. G., Stability of Solutions of Differential Equations in Banach Space, Translations of Mathematical Monographs, vol. 43, American Mathematical Society, Providence, R. I., 1974.

[6] Gil', M. I., Operator Functions and Localization of Spectra, Lecture Notes in Mathematics, vol. 1830, Springer-Verlag, Berlin, 2003.

[7] Gil', M. I., Localization and Perturbation of Zeros of Entire Functions, Lecture Notes in Pure and Applied Mathematics, 258, CRC Press, Boca Raton, FL, 2010.

[8] Gil', M. I., Bounds for the spectrum of a matrix differential operator with a damping term, Z. Angew. Math. Phys. 62 (2011), no. 1, 87-97.

[9] Gohberg, I. C., Krein, M. G., Introduction to the Theory of Linear Nonselfadjoint Operators, Translations of Mathematical Monographs, vol. 18, American Mathematical Society, Providence, R.I., 1969.

[10] Gohberg, I. C., Krein, M. G., Theory and Applications of Volterra Operators in Hilbert Space, Translations of Mathematical Monographs, vol. 24, American Mathematical Society, R. I., 1970.

[11] Krein, S. G., Linear Differential Equations in Banach Space, Translations of Mathematical Monographs, vol. 29, American Mathematical Society, Providence, R.I., 1971.

[12] Kunstmann, P. C., Weis, L., Maximal $L_{p}$-regularity for parabolic equations, Fourier multiplier and $H^{\infty}$-functional calculus, in: Functional Analytic Methods for Evolution Equations, Lecture Notes in Mathematics, vol. 1855, Springer, Berlin, 2004, 65-311.

[13] Rofe-Beketov, F. S., Kholkin, A. M., Spectral Analysis of Differential Operators. Interplay between spectral and oscillatory properties, World Scientific Monograph Series in Mathematics, 7, World Scientific Publishing Co. Pte. Ltd., Hackensack, NJ, 2005.

[14] Yakubov, S., Yakubov, Ya., Differential-Operator Equations. Ordinary and Partial Differential Equations, Chapman and Hall/CRC Monographs and Surveys in Pure and Applied Mathematics, 103, Chapman \& Hall/CRC, Boca Raton, FL, 2000.

Michael Gil'

Department of Mathematics

Ben Gurion University of the Negev

P.O. Box 653, Beer-Sheva 84105

Israel

e-mail: gilmi@bezeqint.net

Received March 30, 2011 